\title{
A Robust Structural Fingerprint Restoration
}

\author{
M. Hassan Ghassemian Yazdi \\ Intelligent Signal Processing Research Center \\ Department of Electrical Engineering, Tarbiat Modares University \\ P.O. Box: 14155-4838, Tehran, Iran
}

\begin{abstract}
Fast and accurate segmentation of fingerprints is essential to each AFIS (Automatic Fingerprint Identification System). Smudged furrows and cut ridges in the image of a fingerprint is the major problem in any AFIS. This paper investigates a new on-line ridges detection method that reduces the complexity and costs associated with the fingerprint identification procedure. A new structural algorithm for restoration of the ridges is described. This algorithm is based on unsupervised fuzzy classification technique. With no cost of time, some new features, such as direction of ridges, have been extracted. The accuracy and speed of the proposed method is tested for a large number of fingerprint images with different initial qualities, and is found to be excellent compared to the conventional methods. The results show a significant improvement in identification/verification performance.
\end{abstract}

\section{Introduction}

Fingerprints have been used as a positive human identifier for more than a century. It is considered one of the most reliable means of distinguishing a man from his fellows. The unchangeability of fingerprints during the human life span and the uniqueness of each individual's fingerprints are the basis for using fingerprints for identification purposes [1]. Fingerprint identification is one of the few scientific aids to investigation of crimes, and it is extensively used in day to day crime investigations. The fingerprint matching technique and the matching results are acceptable to the court of law as conclusive proof of the identity of a person. Court requires a minimum of $8 / 16$ common minutiae for accepting the identity [2]. There are some other application for AFIS: access control (securing area), transaction control (banking and credit card security), production processing .

With the population explosion and the increase in the crime rate, there is an urgent need to develop an automatic fingerprint recognition system to improve the efficiency and reliability of personal identification. The aim of all fingerprinting systems is to produce a low-cost system that is reliable, fast and accurate. In general, the function of fingerprinting systems can be separated into several distinct operations, which include sensing or reading, image processing, print registration, classification search, comparison, and verification. More powerful and intelligent image processing techniques are possible by recent developments in computer technology. This technology is being used to automate the fingerprint identification procedure. A fingerprint could be identified by the shape of ridge or furrow lines and their end points in the fingerprint image, Fig. 1. In general, the fingerprints are irregular due to imperfections such as: ridge gaps caused by skin fold, touching of ridges caused by spreading of ink by finger pressure or excessive inking or by smearing during rolling of fingers. Moreover the fingerprint size is usually small 

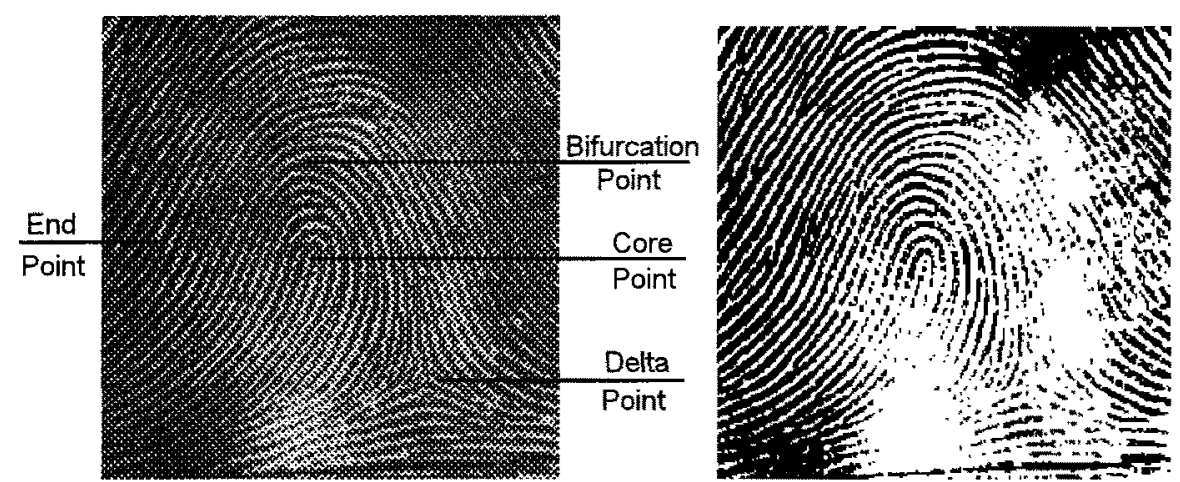

Fig. 1. Common minutiae in a fingerprint. Fig. 2. Segmentation by a Fixed Threshold

and ridges are quite close, so imperfection or noise introduced at any stage of enlarging, and digitization makes the ridge detection more difficult. Enhancement of fingerprint image is necessary where ridges are not very prominent or have nonuniform intensity.The quality of the original print is the major problem in the AFIS. If the quality is not of an acceptable standard, automatic fingerprint identification or verification becomes extremely difficult. With the above consideration in mind, this paper investigates ridges restoration methods that reduces the complexity and costs associated with the fingerprint identification procedure. Following this introduction, section two introduces the main published approaches, and, investigates some basic image segmentation techniques on fingerprints. In section three, we introduce our approach to fingerprint segmentation, by pixel classification. The approach is based on unsupervised fuzzy classification technique. We have presented the results of experiment and their evaluation in section 4. Experimental results are presented in terms of real fingerprint data to demonstrate the effectiveness of the proposed approach. Finally, in the concluding section, the general application of our method and its possible extensions will be considered. We found out that our approach has the advantage of being simple and having a significant improvement in identification performance.

\section{Segmentation Methods}

In segmentation of a fingerprint image a clear ridge area constitutes the foreground, and any other area (including smudged or noisy regions) constitutes the background. Thus, any pixel of the fingerprint image, $f(x, y)$, could be classified either to ridges, 1 , or to background, $\phi$ :

$$
\Psi(f(x, y)) \in\{\phi, 1\}
$$

A good segmentation method, $\Psi($.$) , reduces the effect of poor quality data and it$ usually results in improved classification performance $[3,4]$. This method should not be sensitive to the contrast in the image, it should detect smudged and noisy regions, it should give consistent results for a variety of images expected by the application, and it should be independent of whether the input image is a segmented image or a raw image. This means that the number of iterations does not effect on the results. 
In this section, some image segmentation techniques that are particularly applicable to fingerprint images will be discussed.

Image segmentation techniques usually aim at an optimal trade off between efficiency and implementation simplicity, according to the user's needs. Basically, two different approaches have been available to segment the fingerprint images: pixel segmentation methods and neighborhood segmentation methods. In the pixel segmentation methods, individual pixel values are manipulated independently of other pixels. In the neighborhood segmentation methods, groups of pixels are operated on either in the space domain or in the spatial-frequency domain. The operations performed can be either linear or nonlinear [5-11].

\subsection{Pixel Segmentation Techniques}

The simplest pixel segmentation method is to perform a thresholding operation using an appropriate global threshold, $\mathrm{TH}$ :

$$
\Psi(f(x, y))=\left\{\begin{array}{lll}
\phi & \text { if } & f(x, y) \leq T H \\
1 & \text { else } & f(x, y)>T H
\end{array}\right.
$$

This technique is very sensitive to smudged and noisy regions. This method can be improved by contrast modification:

$$
g(x, y)=\Gamma(f(x, y)
$$

where $\Gamma($.$) is the contrast manipulation function. In the literature on this subject$ several contrast manipulation technique are introduced, [5]. In those techniques, the histogram is manipulated as a probability density function which is to be made uniform or changed to a per-determined density. A number of histogram transformations are also available, such as: histogram stretching, logarithmic or hyperbolic transforms that are designed to mach the response of the human visual system. These methods can be performed very fast. These are the technique that one normally try first when confronted with a high quality fingerprint images. Figure 2 shows one result of such techniques. This method is improved by using adaptive threshold, which is explained in the next section.

\subsection{Neighborhood Segmentation Techniques}

Neighborhood process can be performed by manipulating groups of pixels in small moving windows across the image. The pixel value in the segmented image is determined by a function operation on several pixel values in the moving window in the original image. This function can be either linear or nonlinear. One such process is adaptive binarization, where, an $\mathrm{M}$ by $\mathrm{M}$ window is placed over each pixel in the image and the average gray level within the window is computed. Then the center pixel is set to white or black depending on whether the value of the pixel is less than or greater than the local average [7].

where

$$
\Psi(f(x, y))=\left\{\begin{array}{lll}
\phi & \text { if } & f(x, y) \leq T H(x, y) \\
1 & \text { else } & f(x, y)>T H(x, y)
\end{array}\right.
$$

$$
T H(x, y)=\frac{1}{M^{2}} \sum_{i=-\frac{M}{2} j=-\frac{M}{2}}^{\frac{M}{2}} \sum_{\frac{M}{2}} f(x-i, y-j)
$$




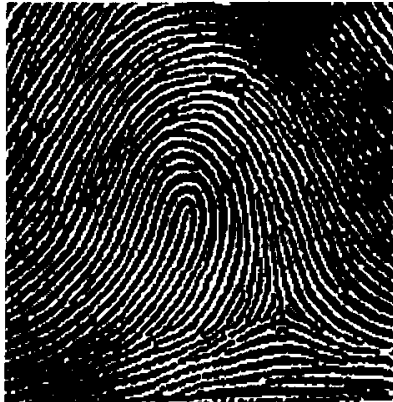

Fig. 3. Adaptive filtering Fig. 4. Marr-Hilderth filter

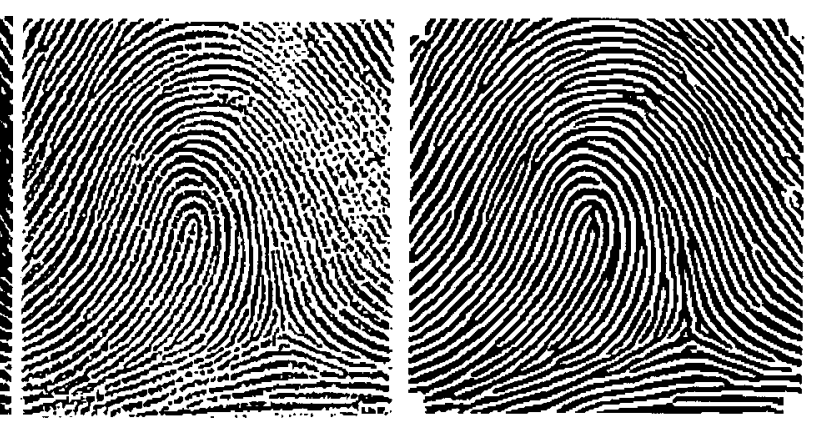

Fig. 5. The proposed technique

Figure 3 shows result of the adaptive binarization algorithm. This technique suffers from noisy regions.

Other example of moving window processing in the spatial domain include edge enhancement for highlighting the ridges. These operation can also be performed in the spatial-frequency domain, such as directional filtering, with less speed but greater flexibility. One of the sophisticated spatial-frequency filter is Laplacian filter. Laplacian filter accentuates the high spatial-frequency terms over the low frequency terms. This has the effect of accentuating edges in the original image while suppressing the slowly varying gray level components. This method is very sensitive to the noisy regions and the wide of ridges [8].

There are several segmentation technique based on edge detection $[9,10]$. These techniques suggest that in order to detect ridges efficiently, one should search for a filter that has two salient characteristics. First and foremost, it should be a differential operator, taking either a first or second spatial derivative of the image. Second, it should be capable of being tuned to act at any desired scale, so that large filters can be used to detect blurry shadow edges, and small ones to detect sharply focused fine detail in the image. Marr and Hildreth argued that the most satisfactory operator fulfilling these conditions is the filter $\nabla^{2} G, \nabla^{2}$ is the Laplacian operator and $G$ stands for the Gaussian distribution which has standard deviation $\sigma . \nabla^{2} G$ is a circular symmetric Mexican-hat-shaped operator whose distribution in two dimensions may be expressed in terms of the radial distance, $r$, by the formula:

$$
\nabla^{2} G(r)=\frac{-1}{\pi \sigma^{4}}\left(1-\frac{r^{2}}{2 \sigma^{2}}\right) e^{\frac{-r^{2}}{2 \sigma^{2}}}
$$

where $r^{2}=x^{2}+y^{2}$. The standard deviation, $\sigma$, is approximately equal to wide of a ridge and a furrow. Then, the segmented image is computed by:

$$
\Psi(f(x, y))=\left\{\begin{array}{lll}
\phi & \text { if } & g(x, y) \leq T H \\
1 & \text { else } & g(x, y)>T H
\end{array}\right.
$$

where, $g(x, y)$ is the convolution of input image and $\nabla^{2} G$ :

$$
g(x, y)=\nabla^{2} G(x, y) * f(x, y)
$$


Figure 4 illustrates the ridges of a fingerprint which are extracted by using the Marr-Hilderth algorithm.

There are two basic ideas behind the choice of the filter $\nabla^{2} \mathrm{G}$. The first is that the Gaussian part of it, G, blurs the image, effectively wiping out all structure at scales much smaller than the space constant $s$ of the Gaussian. The second idea concerns the derivative part of filter, Laplacian operator $\nabla^{2}$. The great advantage of using this operator is economy of computation, also, this operator is orientation independent.

Some of the earlier studies cited above suffered from sudden changes in fingerprint intensity, some from the sensitivity to the noise, and mostly from the limitation due to the necessary conditions requiring large amounts of CPU time, iteration processing, and some from the dependency on manual initiation or supervision for thresholding assignment. In those, time consuming approaches, that the extraction of ridges is based on directional regions, even though they may guarantee ridge connectivity, the extraction of a complex ridge with sudden rotations is not accurate [4]. In the next section, we explain a new segmentation process to solve the above problems.

\section{Segmentation Based on Contextual Features}

In the conventional fingerprint segmentation, a clear fingerprint ridge area constitutes the foreground and any other area constitutes the background. It is desirable that all vague regions such as: the smudged regions and other such noisy regions be included in the background. This paper is concern about restoration of those vague regions in a proper classification process.

Typically, the vagueness in the detection of ridges is caused by smudged furrows and cut ridges in the fingerprint image. A ridge can be distinguished reasonably well on the basis of its intensity feature, using statistical pattern classification techniques. However, the intensity feature of a ridge often has only a weak relation to fingerprint segmentation process. One might expect the segmentation accuracy to be higher by using spectral-contextual feature, rather than when using only the measurement made on the pixels intensity without context.

The orientation pattern in the fingerprint which is fuzzy in nature, and its structural information as a grammar nule, can be incorporated in the segmentation process. Each ridge point, $r$, could be associated with an approximate direction, $\theta_{i}$ :

$$
\theta_{i} \in\left\{\theta_{1}=\pi / 4, \theta_{2}=2 \pi / 4, \theta_{3}=3 \pi / 4, \theta_{4}=\pi\right\}
$$

As we see, this "natural association" has a type of imprecision that stems from a grouping of ridge points into classes that do not have sharply defined directions. Fuzzy set theory is able to model these classes. Such classes arise whenever we describe ambiguity and vagueness in mathematical models of empirical phenomena. Ever since Zadeh introduced the idea of fuzzy set theory by utilizing the concept of membership grade, many researchers have been concerned with the properties and application of fuzzy sets, as can be observed in the literature [12]. 
Let $R=\{r\}$ denote a space of ridge points. Then a fuzzy set $\theta$ in $R$ is a set of ordered pairs:

$$
\theta_{i}=\left\{r, \mu_{\theta i}(r)\right\}, r \in R
$$

where $\mu_{\theta}(r)$ is termed "the grade of membership of $r$ in $\theta$." We shall assume that $\mu_{\theta}(r)$ is a number in the interval $[0,1]$, with the grades 1 and 0 representing, respectively, full membership and non membership in the fuzzy set. It is interesting to note that the grade of membership value $\mu_{\theta}(r)$ of a point $r$ in $R$ can be interpreted as the degree of compatibility of the predicate associated with the direction of ridge $r$. It is also possible to interpret $\mu_{\theta}(r)$ as the degree of possibility that $r$ is the value of parameter fuzzily restricted by $\theta$.

The assignment of the membership function of a fuzzy set is subjective in nature and, in general, reflects the context in which the problem is viewed. But, although the assignment of the membership function of the fuzzy set $R$ is "subjective," it cannot be assigned arbitrarily. The ambiguity in the ridge detection process can be reduced if the spatial dependencies, which exist among the adjacent pixels, are intelligently incorporated into the membership function. We define the unity relation that must exist among the pixels of a ridge. The unity relation can be constructed with regard to the: adjacency relation, spectral-feature and spatial feature characteristics in a ridge. Based on this assumption, we introduce a new membership function by:

$$
\mu_{\theta_{i}}(r)=\lambda_{r}\left(e^{-\frac{\alpha}{2} r^{2}\left(\frac{\pi}{2}-\theta_{i}\right)}\right)\left(1-\alpha r^{2}\left(\theta_{1}\right)\right)\left(e^{-\alpha r^{2}\left(\theta_{i}\right)}\right) \quad i=1,2,3,4 .
$$

where, $\lambda_{r}$ is a normalized factor such that the membership function $\mu_{\theta i}(r) \in[0,1]$, and $\alpha^{-1}$ is approximately equal to wide of a ridge plus a furrow. The reason we chose the Gaussian shape for membership function, is that the Gaussian distribution has the desirable characteristic of being smooth and localized in both the spatial and frequency domains. In strict sense, it has a unique distribution in spatial and frequency domains, which optimally localized in both domains.

We have assumed that an exact comparison is possible for the truths of any two inexact statements " $r_{i} \in R$ " and " $r_{k} \in R$," and that the exact relation so obtained satisfies the minimal consistency requirements of transitivity and reflexivity. The $r_{i}>r_{k}$ means " $r_{i}$ is at least as true as $r_{k}$ " with $r_{i}<r_{k}$ denoting " $r_{i}$ is not truer than $r_{k}$." Based on this assumption, the membership directions of each pixel is computed. Intuitively, $r$ belongs to a class that admits the highest membership in it:

$$
\mu_{\theta i}(r)=\operatorname{Max}\left\{\mu_{\theta k}(r) ; k=1,2,3,4\right\}
$$

Then, the image is segmented by using the natural structure of fingerprint pattern (orientation) as a grammar rule, i.e. a ridge consistency is checked by comparing the membership with its perpendicular direction membership:

if $\mu_{\theta i}(r)-\mu_{\theta i+2}(r)>0.5$ then: $\Psi\left\{r_{i}\right\}=1$, else: $\Psi\left\{r_{i}\right\}=\phi$

In the case of $\Psi\left\{r_{i}\right\}=\phi$ : this pixel belongs to background valleys, in the other case, $\Psi\left\{r_{i}\right\}=1$, it belongs to a ridge. Figure 5 depicts the result of segmentation by the above procedure. This image has additional information about the ridges direction which can be used as a new feature for fingerprint identification. The direction of this ridge point, $\theta_{\mathbf{r}}$, is computed by the following simple algorithm: 


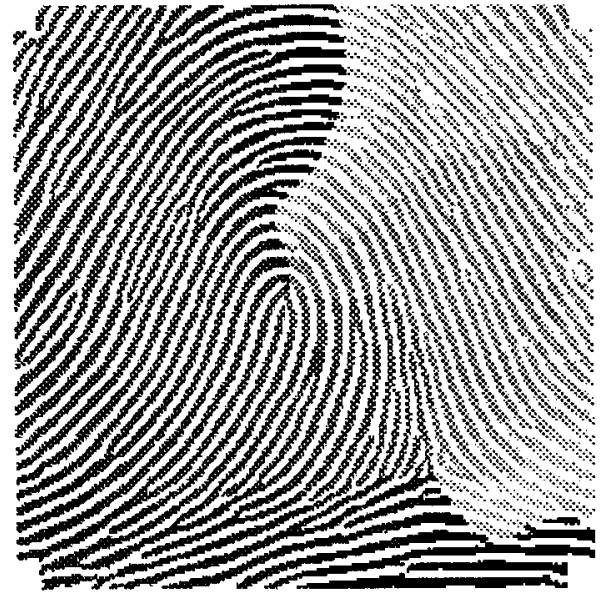

Fig. 6. Direction of ridges

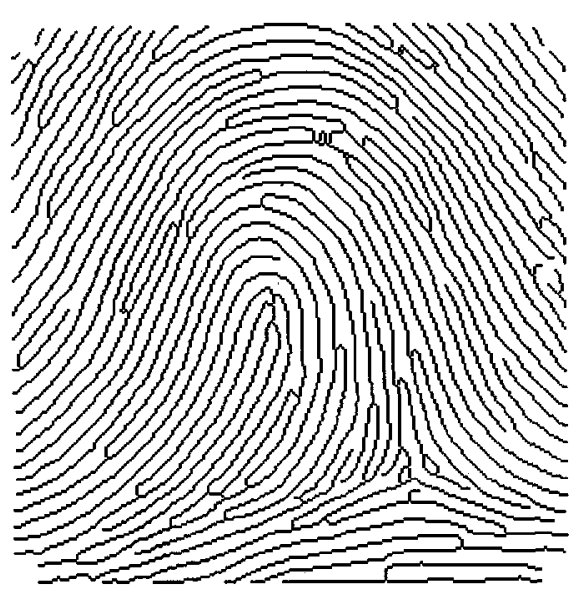

Fig. 7. Example result of thinning algorithm

if $\mu_{\theta i}(r)-\mu_{\theta i+1}(r)<0.5$ then: $\theta_{r}=\theta_{i}+\pi / 8$,

else if $\mu_{\theta i}(r)-\mu_{\theta i-1}(r)<0.5$ then: $\theta_{r}=\theta_{i}-\pi / 8$, else $\theta_{r}=\theta_{i}$

where $\theta_{\mathrm{r}} \in\{\pi / 8,2 \pi / 8,3 \pi / 8,4 \pi / 8,5 \pi / 8,6 \pi / 8,7 \pi / 8, \pi\}$, Figure 6 depicts the result of ridges' direction classification.

\section{Results}

A program which implements the procedures described in this work, was written in $\mathrm{C}$ and run on an IBM compatible PC with 80486 DX4 processor. The CPU time for each fingerprint is less than 0.5 second. Experimental results are presented in terms of real fingerprint data to demonstrate the effectiveness of the proposed approach. Examples show good ridge enhancement and noise reduction. The accuracy and the speed of this method is tested for a large number of fingerprint images with different initial qualities and is found to be excellent compared with some other methods. The segmented image has additional information about the ridges direction, which can be used as a new feature for fingerprint identification system. Using the ridges direction, we developed an extremely fast, on-line, and accurate algorithm for thinning the fingerprint images. The result of this algorithm is depict in Fig. 7. This algorithm has been used for an on line fingerprint verification system. The system has a significant improvement in speed and performance [13].

\section{Summary and Conclusion}

We discussed the reliability in application of fingerprints for individual identification in a security system. We explained the main sources of miss recognition in an automatic fingerprint identification system. To realize this system the first problem to be solved is the acquisition of a high quality image of the fingerprint.

In this paper we discussed noise elimination and restoration of ridges, which should be incorporated in the preprocessing operation of an automatic fingerprint 
identification system. In other words, a robust segmentation is the first step toward further process, leading to efficient encoding, storage, and recognition of a fingerprint.

An unsupervised fuzzy classification technique is used for fingerprint segmentation. We introduced a new membership function, by using the property of oriented pattern in the fingerprints, which is fuzzy in nature. Also, the structural information of finger patterns, as a grammar rule, is incorporated in the process to enhance the segmented image.

The procedure is on line, unsupervised and adaptive. Examples show good ridge enhancement and noise reduction. The accuracy and the speed of this method is tested for a large number of fingerprint images with different initial qualities and is found to be excellent compared with some other methods. The resulting images are enhanced, noise reduced, and binary.

The segmentation procedure is of course a first, but important, step in a fingerprint recognition system. Nevertheless, we feel that other steps, such as feature selection and classifier design, as well as the first step, can improve the cost and performance of the AFIS. We found out that, the segmented image has additional information about the ridges direction, which can be used as a new feature for fingerprint identification system.

\section{References}

[1] B. Miller, "Biometrics: Vital Signs of Identity," IEEE Spectrum, pp. 22-30, (1994).

[2] B.C. Bridges, Practical Fingerprinting, Furank \& Wagnalls Co. (1942).

[3] G. Candella and R. Chellappa, "Comparative Performance of Classification Methods for Fingerprints," National Institute of Standards and Technology, April (1993).

[4] M.H. Ghassemian, "Automatic Singular Points Detection in the Fingerprints," Proc. of ICEE-94, Vol.5, pp.286-294, (1994)

[5] R.M. Haralick, L.G. Shapiro, "Image Segmentation Techniques," Computer Vision, Graphics an Image Processing, vol. 29, pp. 100-132, (1986).

[6] T.N. Pappas, "An Adaptive Clustering Algorithm for Image Segmentation," IEEE Trans. on Signal Processing, vol. 40, no. 4, pp. 901-914, (1992).

[7] P.W. Palumba, et al, "Document Image Binarization, Evaluation of Algorithms," SPIE, Vol.697, Application of Digital Image Processing DX, pp. 278-285, (1986).

[8] T.F. Krile, J.F. Walkup, "Enhancement of Fingerprints Using Digital and Optical Techniques," Image Analysis Application, Marcel Dekker, pp. 343-371, (1990).

[9] S. Ghosal and R. Mehrotra, "Detection of Composite Edges," IEEE Trans. Image Processing, vol. 3, no. 1, Jan. (1994).

[10] C.C. Chue and J.K. Aggarwal, "The Integration of Image Segmentation Maps Using Region and Edge Information," IEEE Tran. PAMI (Pattern Analysis and Machine Intelligence), vol. 15, no. 12, Dec. (1993).

[11] B.M. Mehtre and B. Chatterjee, "Segmentation of Fingerprint Images A Composite Method," Pattern Recognition, Vol.22, No.4, pp. 381-385, (1989).

[12] A. Kandel, Fuzzy Techniques in Pattern Recognition, Wiley, (1982).

[13] L. Lam, et al, "Thinning Methodologies a Comprehensive Survey," IEEE Tran. on PAMI, vol. 14, no. 9, pp. 869-885, (1992). 